Objet : Rétractation de I'article DOI : 10.19182/remvt.36815

L'article suivant :

Bouziri, A., Sebai, A., Kebir, A., Khames, M., Hamdi, T. M. Khelef, D., 2021. Fièvre aphteuse chez les bovins et les petits ruminants en Algérie. Enquête séroépidémiologique dans la région de l'ouest, Rev. Elev. Med.Vet. Pays Trop., 74 (4) : 225-230. doi: 10.19182/remvt.36815

a été rétracté le 11 avril 2022, sur demande des auteurs et avec l'accord des rédacteurs en chef.

Le motif de rétractation est :

Manque de fiabilité des données présentées du fait des protocoles utilisés pour leur production, selon des informations qui ont été connues après la publication de l'article.

Les données présentées dans cet article ne peuvent donc plus être considérées comme valides et ne doivent pas être utilisées ou citées.

L'article est cependant laissé en ligne aux fins de traçabilité, accompagné de la présente notice et de l'indication de la rétractation sur toutes les pages du fichier.

Les rédacteurs en chef 
Re: Article Retraction - DOI: 10.19182/remvt.36815

The following article:

Bouziri A., Sebai A., Kebir A., Khames M., Hamdi T.M., Khelef D., 2021. Foot-and-mouth disease in cattle and small ruminants in Algeria. Seroepidemiological survey in the Western region. Rev. Elev. Med. Vet. Pays Trop., 74 (4): 225-230, DOI: 10.19182/remvt.36815

was retracted on April 11, 2022, at the Authors' request and in agreement with the Editors in Chief.

The reason for retraction is:

Unreliability of the data presented due to the protocols used to produce them, based on information that became known after publication of the article.

The data presented in this article can no longer be considered valid and should not be used nor cited.

However, for traceability purposes the article is kept online along with this note, and the mention of retractation has been stamped across each page.

The Editors in Chief 
REPUBLIQUE ALGERIENNE DEMOCRATIQUE ET POPULAIRE

MINISTERE DE L'AGRICULTURE ET

DU DEVELOPPEMENT RURAL

INSTITUT NATIONAL DE LA MEDECINE

VETERINAIRE

Rue Ouled Sidi Cheikh Mohammadia Alger

BP 205 Hacen Badi El Harrach Alger

(+213) 23820990

Email: inmv@madr.gov.dz

Réf : $9.52 .0 G$ IINMV
وزارة الفـلاحة و التنمية الـريفية

المعهد الوطني للطب البيطري

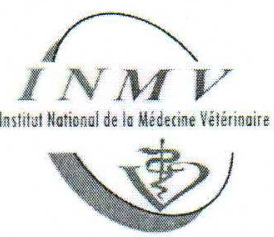

Alger le, ........6...A4R: 2022

\section{A Monsieur le responsable de la publication}

\section{Revue élevage et médecine vétérinaire des pays tropicaux}

Suite à la publication de l'article intitulé «Fièvre aphteuse chez les bovins et les petits ruminants. Épidémiologique dans la région de l'Ouest» dans votre revue volume 74 $N^{\circ} 04$ (2021), j'ai l'honneur de vous apporter la mise au point suivante :

- Le laboratoire Vétérinaire Régional de Mostaganem est un laboratoire dépendant de I'Institut national de la Médecine Vétérinaire, sous tutelle du Ministère de l'Agriculture et du Développement Rural, dont le siège est à Alger.

- Le travail objet de votre article n'a pas été réalisé par les personnes nommées, ces dernières ne sont pas habilitées à faire des analyses de virologie dans nos services.

- Le Dr Abdeldjalal Bouziri et le Dr Ali Sebai exercent dans le service de bactériologie médicale et n'ont ni effectué ces analyses ni géré l'équipe de technicien chargé de l'analyse.

- Le Dr Ahmed Kebir n'a jamais réalisé les prélèvements sanguins de cette enquête, ni collecté les données.

- Les prélèvements analysés au niveau du laboratoire de Mostaganem dans le cadre de cette enquête épidémiologiques n'ont pas fait l'objet d'un prétraitement pour écarter les faux positifs dues aux réactions croisées.

- Seuls les sérotype O et A ont été détectés lors des épisodes de 2014, 2017 et 2018.

- Lors de ces épisodes des prélèvements ont été envoyés au laboratoire de référence de l'OIE et ce dernier a toujours confirmé la présence de serotypes $O$ et $A$, d'où l'utilisation d'un vaccin bivalent $O$ et $A$.

Aussi je vous saurais gré de publier cette mise au point pourr éclaixer vos lecteurs.

Veuillez recevoir Monsieur, l'expression de salutations respectueuses.

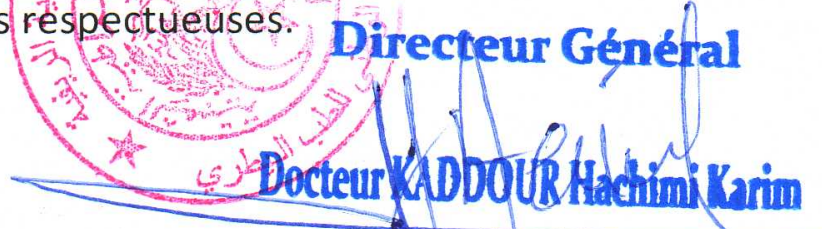




\title{
Fièvre aphteuse chez les bovins et les petits ruminants en Algérie. Enquête séroépidémiologique dans la région de l'ouest
}

\author{
Abdeldjalal Bouziri ${ }^{1,2 *}$ Ali Sebai ${ }^{2}$ Ahmed Kebir ${ }^{2}$ \\ Maamar Khames ${ }^{4}$ Taha Moussadak Hamdi ${ }^{3}$ Djamel Khelef
}

Mots-clés

Bovin, petits ruminants, fièvre aphteuse, morbidité, Algérie

(C) A. Bouziri et al., 2021

\section{(c) (1)}

https://creativecommons.org/licenses/by/4.0/

Submitted: 14 February 2021

Accepted: 27 October 2021

Published: 20 December 2021

DOI: $10.19182 /$ remvt.36815

\section{Résumé}

La fièvre aphteuse (FA) est une maladie infectieuse d origine virale qui affecte les ruminants et les porcins. Une enquête seroépidémio dgique a été menée chez les ruminants de la région ouest de l'Algérie afin d'estimer la séroprévalence de la FA et de déterminer les facteurs de risque susceptibles de favoriser l'atteinte des animaux par cette pathologie. Au total 420 sérums ont été collectés (210 de bovins et 210 de petits ruminants) et soumis au test Elisa NSP permettant la détection des anticorps dirigés contre le vi us de la FA induits par les protéines non structurales, puis à des tests Elisa détectant les protéines structurales spécifiques des sérotypes A, O, SAT 1, SAT 2 et Asia 1. Les résultats ont montré une différence non significative $(p>0,05)$ de la séroprévalence entre les especes avec $23,8 \%$ chez les bovins et $27,1 \%$ chez les petits ruminants. Le sérotype O était présent dans 95,3\% des sérums positifs. Il était present seul chez $649 \%$ des petits ruminants alors que les bovins étaient généralement inféctés par plusieurs sérotypes simultanément, avec l'association prédominante des quatre sérotypes A, O, SAT 1 et Asia 1, qui représentait $36 \%$ des infections. Le taux de couverture vaccinale a atteint $48,7 \%$ du cheptel (bovins et petits ruminan's) âgé de plus de six mois. Aucun lien statistique n'a été mis en évidence entre le mode d'élevage (extensif, semi-intensif ou intensif) et l'atteinte a la FA' $p>0,05)$. En revanche, une association significative $(p<0,05)$ a été établie entre la vaccination des animaux et l'infection, les animaux vaccinés ayant été moins infectés par la FA.

Comment citer cet article : Bouziri A., Sebai A., Kebir A., Khames M., Hamdi T.M., Khelef D., 2021. Foot-and-mouth disease in cattle and small ruminants in Algeria. Seroepidemiological survey in the Western region. Rev. Elev. Med. Vet. Pays Trop., 74 (4): 225-230, doi: 10.19182/remvt.36815

\section{- INTRODUCTION}

La fièvre aphteuse (FA) est une maladie virale transfrontalière très ntagieuse. Tous les animaux artiodactyles domestiques et sauvages y sont sensibles (Leforban, 2003). L'Organisation mondiale de la anté animale (OIE) a classé la FA parmi les maladies à notification

1. Laboratoire de Santé et production animale (SPA), Ecole nationale supérieure vétérinaire Rabie Bouchama, rue Issad Abbes, Oued Smar-Alger, Algérie.

2. Laboratoire vétérinaire régional de Mostaganem (LVRM), Hassi Mameche, Mostaganem.

3. Laboratoire HASAQ, Ecole nationale supérieure vétérinaire d'Alger (ENSV).

4. Université de Yahia Farès de Médéa.

* Auteur pour la correspondance

Tél. : +213 (0)6 66432042 ; email : bouziri.vet@ gmail.com obligatoire en raison de sa diffusion potentiellement rapide et pouvant s'étendre sur plusieurs pays, et des pertes économiques considérables qu'elle engendre sur la production animale et en entravant le commerce international des animaux et des denrées d'origine animale (Alexandersen et al., 2003). Selon les estimations de l'OIE, cette maladie circule au sein de $77 \%$ du bétail mondial, en Afrique, au Moyen-Orient et en Asie, ainsi que dans une zone restreinte d'Amérique du Sud (OIE, 2018b). Au cours des dernières années, et particulièrement en 2005-2006, le monde a fait face à une recrudescence de la maladie avec de nombreux foyers affectant des millions d'animaux et occasionnant d'importantes pertes économiques en Asie, en Amérique latine et en Afrique (Houndjè et al., 2013). L'Algérie a également connu plusieurs épisodes de FA durant ces dernières décennies, dont les plus marquants ont été ceux des années 1999, 2014-2015 et 2018, au cours desquels les taux de morbidité et de mortalité les plus élevés 
ont été observés chez les espèces bovines, ovines et caprines. Durant ces épisodes, les sérotypes $\mathrm{A}$ et $\mathrm{O}$ du virus aphteux ont été identifiés (commun. Direction des vétérinaires algériens, 2019). Le virus de la FA présente en effet une grande variabilité génétique puisqu'il existe actuellement sept sérotypes immunologiques distincts $(\mathrm{O}, \mathrm{A}, \mathrm{C}$, SAT 1, SAT 2, SAT 3 et Asia 1) qui circulent dans le monde.

Lors de l'épidémie de fièvre aphteuse de 2014-2015, l'analyse phylogénétique des isolats viraux a permis d'identifier la souche $\mathrm{O} /$ ME-SA/Ind-2001 sérotype O qui a circulé en 2013 en Libye, en 2014 en Tunisie, et a été introduite en Algérie par la Tunisie. Il s'agissait d'un nouveau sérotype au Maghreb, provenant du Moyen-Orient (Bouguedour, 2017). Lors de l'épidémie de FA de 2018, le sérotype O, topotype East Africa 3, a été identifié (Calavas et al., 2018).

Pour faire face à ces épidémies, l'Algérie a mis en place plusieurs dispositifs. Des campagnes de vaccination des bovins et des petits ruminants contre la FA ont été lancées dès l'apparition des premiers foyers, à l'aide d'un vaccin bivalent A et O (Calavas et al., 2018). Les sérotypes utilisés dans le vaccin doivent en effet correspondre aux types sérologiques des souches isolées sur le terrain. Selon un rapport semestriel des autorités algériennes de 2017, presque deux millions de bovins ont été vaccinés de manière préventive contre la FA de juillet à décembre 2017. D'autres campagnes ont eu lieu en 2018 et 2019, c'està-dire au cours des années précédant notre étude.

L'identification du cheptel national bovin a d'autre part été initiée grâce à un projet de jumelage avec l'Union européenne (2014-2016) en vue du renforcement de la traçabilité et de l'identification des animaux. Enfin, des laboratoires d'alerte précoce (appelés observatoires) ont été installés dans les wilayas (préfectures) frontalières du grand sud, observatoires dont le rôle principal est la détection rapide et le diagnostic précoce des cas de FA transfrontalière. Dans ce contexte. les objectifs de notre étude ont été d'estimer la séroprévalence de la fièvre aphteuse dans le cheptel de bovins et de petits ruminants à l'ouest de l'Algérie, où se situe le laboratoire ayant réalisé les analyses, d'identifier les facteurs de risque d'infection, de caractériser les séro types des souches de virus de la FA circulant au sein des élevages, et d'estimer le taux de couverture vaccinale par le vaccin antr-aphteux.

\section{- MATERIEL ET METHODES}

\section{Site et réalisation de l'étude sur le terrain}

Cette enquête a été menée à l'ouest de l'Algérie 15 au 26 septembre 2019. Compte tenu du nombre de kits Elisa disponibles, 420 prélèvements sanguins (210 de bovins et 210 de petits ruminants) ont été effectués dans sept wilayas (Mostaganem, Oran, Mascara, Tiaret, Chlef, Relizane et Tissemsilt, figure 1), à raison de 30 prélèvements par espèce et par wilaya répartis dans trois élevages différents. La sélection des élevages visités a été effectuée par tirage aléatoire à partir des listes disponibles d'élevages de chaque wilaya. Si l'élevage sélectionné comptait moins de 10 animaux, l’échantillonnage était complété par un autre élevage du même site. Les animaux à prélever ont également été sélectionnés de manière aléatoire.

Les prélèvements sanguins ont été réalisés à la veine caudale chez les bovins et à la veine jugulaire chez les petits ruminants dans des tubes secs de $5 \mathrm{ml}$. Ils ont été transportés sous couvert du froid jusqu'au Laboratoire vétérinaire régional de Mostaganem (LVRM) où ils ont été centrifugés à 3500 tours/min pendant 5 min en vue de leur analyse.

Parallèlement, un questionnaire a été rempli pour chaque élevage ayant fait l'objet de prélèvements. Des informations relatives au mode d'élevage (intensif, semi-intensif ou extensif), au type de production (laitier, reproduction, engraissement) et à la vaccination du cheptel vis-à-vis de la FA ont été recueillies. Cette vaccination était effectuée sur tous les animaux de plus de six mois lors de campagnes annuelles qui avaient lieu chaque année depuis 2017

\section{Analyses de laboratoire}

Les analyses ont été effectuées auLVRM par la technique immunoenzymatique (Elisa) en utilisant la gamme de kits commerciaux PrioCHECKR. Dans un premier temps, tous les sérums ont été soumis au test Elisa recherchant des anticorps dirigés contre les protéines non structurales (NSP) du irus de la FA, protéines qui ne sont synthétisées que lors de la multiplication du virus. Ce test permet de distinguer les anticorps généés par l'infection de ceux induits par la vaccination (Leforban, 2003). Les NSP peuvent ainsi être considérées comme des indicateurs de l'infection indépendamment du statut vaccinal de l'animal (OIE, 2018c). Seuls les animaux positifs à ce test sont par la suite considérés comme séropositifs.

Puis les sérums positifs au test Elisa NSP ont été soumis aux divers tests Elisa caractéristiques des types A, O, SAT 1, SAT 2 et Asia 1 afin d'identifier les sérotypes en cause par la recherche des anticorps dirigés contre les protéines structurales spécifiques du virus aphteux. Les sérotypes SAT 3 et C n'ont pas été inclus dans l'enquête car, d'une part, les kits n'étaient pas disponibles, d'autre part, ces sérotypes rares ne circulent pas dans les pays qui entourent l'Algérie. Le test est de type bloquant, basé sur une compétition entre les anticorps monoclonaux et les anticorps présents dans l'échantillon.

Chaque technique Elisa a été réalisée selon la notice fournie par le fabricant en respectant les recommandations des normes Afnor NF-U47-019 de février 2010 ( $«$ Guide de bonnes pratiques pour la mise en œuvre des techniques Elisa ») et NF-U47-020 de juillet 2001 (« Guide de bonnes pratiques de traitement de l'échantillon soumis à des analyses immunosérologiques »).

\section{Analyses statistiques}

La comparaison de la séroprévalence (taux d'animaux ayant été infectés par la FA) et des pourcentages de positivité des sérotypes A, O, SAT 1, SAT 2 et Asia 1 entre les bovins et les petits ruminants, et

Figure 1 : Répartition des foyers de fièvre aphteuse des bovins et des petits ruminants dans I'Ouest algérien //I Distribution of foot-and-mouth disease outbreaks in cattle and small ruminants in the study area in Western Algeria 
l'association entre le statut sérologique des animaux et les variables « mode d'élevage » (extensif, intensif et semi-intensif) et « vaccination » ont été effectuées par le test de $\mathrm{Chi}^{2}$. Les tests ont été réalisés avec le logiciel Xlstat version 2015. Le rapport de cote (odds ratio) a été calculé pour évaluer le degré de dépendance entre le statut vaccinal, connu par le questionnaire, et le statut sérologique. Les différences ont été considérées comme significatives pour $\mathrm{p} \leq 0,05$.

\section{RESULTATS}

Sur les 420 sérums soumis au test Elisa NSP, 107 ont été trouvés séropositifs soit une séroprévalence globale de $25,5 \%$ (intervalle de confiance à $95 \%$ [IC95] : 21,3-29,6\%). Parmi ces sérums positifs, 50 provenaient de bovins et 57 de petits ruminants, soit des séroprévalences respectives de $23,8 \%$ et $27,1 \%$ (pas de différence significative $: p=0,43$ )

Les tests de sérotypage réalisés sur les 107 sérums positifs ont permis l'identification des cinq sérotypes et ont montré la prédominance marquée du sérotype $O$ présent dans 102 sérums (prévalence observée : 95,3 \% ; IC95 : 91,3-99,3\%). Il y avait également 53 sérums positifs au sérotype A (49,5\% ; IC95 : 40,1-59,0 \%), 38 au sérotype SAT 1 (35,5\% ; IC95 : 26,4-44,6\%), 35 au sérotype Asia 1 (32,7\% ; IC95 : 23,8-41,6\%) et 3 au sérotype SAT 2 (2,8\% ; IC95 : 0,0-5,9\%) (tableau I). Un seul sérotype (A ou O) a été identifié dans 47 des 107 sérums $(43,9 \%)$ soumis au sérotypage, deux ou trois sérotypes dans 19 sérums (17,8\%), quatre sérotypes dans 21 sérums $(19,6 \%)$, et les cinq sérotypes dans un seul sérum $(0,9 \%)$.

Sur les 57 sérums séropositifs chez les ovins et caprins, 37 (64,9\%) étaient positifs uniquement pour l'antigène $O$. D'autre part, 9 sérums $(15,8 \%)$ contenaient d'autres sérotypes que les trois majoritairemen présents, A, O ou SAT 1. Chez les bovins, c'est l'association des quatre sérotypes A, O, SAT 1 et Asia 1 qui était prédominante, avec $36 \%$ des infections, le sérotype $\mathrm{O}$ seul étant présent dans $16 \%$ de sérums. Les sérotypes $\mathrm{A}$ et $\mathrm{O}$ ont été retrouvés seuls ou conjointement, en l'absence d'autres types, dans 58 sérums des bovins et petits ruminants, soit 54,2\% des sérums positifs (tableau II). Autrement dit, $45,8 \%$ des sérums contenaient au moins un sérotype non prêsent dans les vaccins. Ces sérums représentaient 24,6\% des échantillons des petits ruminants et 70,0\% des échantillons bovins $(\mathrm{p}<0,001)$.

Le sérotype $\mathrm{O}$ a été retrouvé dans 54 des 57 sérums positifs de petits ruminants $(94,7 \%)$ et dans 48 des 50 sérums positifs de bovins $(96,0 \%$ ) (différence non significative ; $\mathrm{p}>0$ 05). Par contre, le sérotype A a été retrouvé dants $26,3 \%$ des sérums positifs de petits ruminants et dans 76,0 \% des sérums bovins positifs, Asia 1 respectivement dans $12,3 \%$ ovins-caprins) et 56,0\% (bovins) des échantillons positifs, et SAT $1 /$ dans 17,5\% (ovins-caprins) et 56,0 \% (bovins) (différences signilícatives dans ces trois cas ; $p<0,05)$. SAT 2 a présenté le pourcentage de positivité le plus faible : $3,5 \%$ des petits ruminants et $2 \%$ des boyins (différence non significative ; $p>0,05$ ).

ableau I: Répartition des sérotypes de virus de la fièvre aphteuse identifiés en fonction de l'espèce animale dans I'Ouest algérien /II Distribution of identified foot-and-mouth disease virus serotypes cording to animal species (Western Algeria)

\begin{tabular}{|c|c|c|c|c|c|c|c|}
\hline & \multirow{2}{*}{$\begin{array}{l}\mathrm{Nb} \text {. de } \\
\text { sérums } \\
\text { positifs }\end{array}$} & \multicolumn{6}{|c|}{ Sérotypes identifiés } \\
\hline & & A & $\mathrm{O}$ & SAT 1 & SAT 2 & Asia 1 & Total \\
\hline Bovins & 50 & 38 & 48 & 28 & 1 & 28 & 143 \\
\hline $\begin{array}{l}\text { Petits } \\
\text { ruminants }\end{array}$ & 57 & 15 & 54 & 10 & 2 & 7 & 88 \\
\hline Total & 107 & 53 & 102 & 38 & 3 & 35 & 231 \\
\hline
\end{tabular}

Au moins un animal séropositif a été identifié dans chacune des wilayas avec des taux de séroprévalence variables d'une wilaya à l'autre (tableau III ; figure 1). Tissemsilt et Relizane ont enregistré les séroprévalences les plus élevées avec respectivement $36,6 \%$ chez les bovins à Relizane et 46,6\% chez les petits ruminants à Tissemsilt. Par contre, Chlef et Mostaganem ont affiché les séroprévalences les plus faibles pour les bovins et les petits ruminants avec un taux de $3,3 \%$ chacune.

Le mode d'élevage semi-intensif était le plus répandu (57,1\% des 42 élevages) parmi les troupeaux enquêtés, suivi du mode extensif $(23,8 \%)$ et intensif $(19,0 \%)$. Toutefois, le type d'élevage n'a pas eu d'influence sur la séroprévalence $(\mathrm{p}=0,589)$ (tableau $\mathrm{IV}$.

Parmi les 420 animaux testés, 230 étaient âgés de plus de six mois. Parmi eux, seuls 112 animaux avaient été vaccinés eontre la FA (tableau V), soit un taux de couverture vaccinale des animaux de plus de six mois de $48,7 \%$. Une plus faible proportion des animaux vaccinés $22,3 \%$ contre $48,3 \%$ ) ont été trouvés séropositifs avec le testElisa NSP, donc infectés par le virus $(\mathrm{p}<0,001)$. Il y avait aimsi une association positive entre la vaccination et la prévention contre la fièvre aphteuse.

Tableau II : Fréquence des sérotypes rencontrés chez les ruminants séropositifs à l'Elisa NSP (recherchant les anticorps dirigés contre les protéines non structurales du virus aphteux) dans I'Ouest algérien //I Frequency of serotypes found in ruminants seropositive to NSP ELISA (searching tor antibodies directed against nonstructural proteins of the foot-and-mouth disease virus) in Western Algeria

\begin{tabular}{lcc}
\hline Sérotypes & Petits ruminants & Bovins \\
\cline { 2 - 3 } & $\mathbf{n}(\%)$ & $\mathbf{n}(\%)$ \\
\hline A, O, SAT 1 & $37(64,9)$ & $8(16,0)$ \\
O, Asia 1 & $4(7,0)$ & $7(14,0)$ \\
A, O, SAT 1, Asia 1 & $4(7,0)$ & $4(8,0)$ \\
A & $3(5,3)$ & $0(0)$ \\
A, O, SAT 2 & $2(3,5)$ & $18(36,0)$ \\
A, O, SAT 1, SAT 2 & $2(3,5)$ & $0(0)$ \\
O, SAT 1 & $1(1,8)$ & $0(0)$ \\
A, SAT 1, Asia 1 & $1(1,8)$ & $0(0)$ \\
O, SAT 1, Asia 1 & $1(1,8)$ & $3(6,0)$ \\
A, O, SAT 1, SAT 2, Asia 1 & $1(1,8)$ & $1(2,0)$ \\
A, O, Asia 1 & $1(1,8)$ & $1(2,0)$ \\
A, Asia 1 & $0(0)$ & $1(2,0)$ \\
& $0(0)$ & $6(12,0)$ \\
\end{tabular}

Tableau III : Séroprévalence de la fièvre aphteuse selon la wilaya dans I'Ouest algérien /// Foot-and-mouth disease seroprevalence by wilaya in Western Algeria

\begin{tabular}{lcc} 
Wilayas & Petits ruminants & Bovins \\
\cline { 2 - 3 } & $\mathbf{n}(\mathbf{\%})$ & $\mathbf{n}(\%)$ \\
\hline Tissemsilt & $14 / 30(46,6)$ & $8 / 30(26,6)$ \\
Relizane & $10 / 30(33,3)$ & $11 / 30(36,6)$ \\
Chlef & $9 / 30(30,0)$ & $1 / 30(3,3)$ \\
Mascara & $9 / 30(30,0)$ & $4 / 30(13,3)$ \\
Oran & $8 / 30(26,6)$ & $8 / 30(26,6)$ \\
Tiaret & $6 / 30(20,0)$ & $8 / 30(26,6)$ \\
Mostaganem & $1 / 30(3,3)$ & $10 / 30(33,3)$ \\
Total & 27,1 & 23,8
\end{tabular}


Tableau IV : Influence du mode d'élevage sur la séropositivité des ruminants au virus de la fièvre aphteuse dans l'Ouest algérien //I Influence of the farming system on the seropositivity of ruminants to the foot-and-mouth disease virus in Western Algeria

\begin{tabular}{lccr} 
Mode d'élevage & Séropositifs & Séronégatifs & Total \\
\cline { 2 - 3 } & $\mathbf{n}(\%)$ & $\mathbf{n}(\%)$ & \\
Intensif $(\mathrm{n}=8)$ & $21(26,3)$ & $59(73,8)$ & 80 \\
Semi-intensif $(\mathrm{n}=24)$ & $57(23,8)$ & $183(76,3)$ & 240 \\
Extensif $(\mathrm{n}=10)$ & $29(29)$ & $71(71)$ & 100 \\
Total $(\mathrm{n}=42)$ & 107 & 313 & 420
\end{tabular}

Tableau V : Influence du statut vaccinal * sur la séropositivité des ruminants au virus de la fièvre aphteuse dans l'Ouest algérien //I Influence of vaccination status* on seropositivity of ruminants to foot-and-mouth disease virus in Western Algeria

\begin{tabular}{lccc} 
& Séropositifs & Séronégatifs & Total \\
\cline { 2 - 3 } & $\mathbf{n ~ ( \% )}$ & $\mathbf{n ~ ( \% )}$ & \\
\cline { 2 - 3 } Vaccinés & $25(22,3)$ & $87(77,7)$ & 112 \\
Non vaccinés & $57(48,3)$ & $61(51,7)$ & 118 \\
Total & $82(35,7)$ & $148(64,3)$ & 230
\end{tabular}

* Seuls les animaux de plus de six mois $(n=230)$ sont susceptibles d'être vaccinés en Algérie /// Only animals over six months of age $(n=230)$ are eligible for vaccination in Algeria

révalences estimées lors de cette enquête ont été de $23.8 \%$ chez les bovins et de 27,1\% chez les petits ruminants. Ceci indique l'importance de la circulation du virus aphteux en Algérie, confirma le statut non indemne du pays pour la FA. La dernière notification de foyers de FA en Algérie a d'ailleurs été introduite àl'OIE en juin 2018 (OIE, 2018a). Actuellement, l'OIE ne reconnaît que trois catégories de pays en ce qui concerne la FA (Maree et al., 2014) : les pays indemnes de FA où la vaccination n'est pas pratiquée (cas des pays de l'Union européenne), les pays indemnes de FA ou est pratiquée la vaccination, et les pays infectés par la FA. En 2014, après 15 ans d'absence de la FA, l'Algérie est passée du staut de pays indemne avec vaccination au statut de pays infecté. Depuis 2018, la FA reste endémique en Algérie malgré les campagnes de vaccination.

La différence de séroprévalence globale entre les bovins et les petits ruminants n'était pas significative, mais il y avait des divergences pour ce qui concernait les sérotypes retrouvés. Les sérotypes $\mathrm{O}$ et SAT 2 affectaient les bovins et les petits ruminants sans prédilection d'espece, ma s les sérotypes A, SAT 1 et Asia 1 étaient retrouvés plus souvent chez les bovins que chez les petits ruminants. Ailleurs dans le monde les bovins sont généralement considérés comme étant le principal réservoir du virus de la FA. Les petits ruminants peuvent jouer un rôle important dans sa propagation mais il n'est pas certain que le virus puisse être maintenu chez ces espèces pendant de longues periodes en l'absence d'infection des bovins (OIE, 2018c).

Des foyers de la FA ont été détectés par séroprévalence dans toutes les wilayas ciblées. Certains élevages participant à l'enquête avaient d'ailleurs été touchés par des épisodes de FA en 2014, 2017 et 2018. La propagation de la maladie est due à l'excrétion massive de virus par les animaux infectés et à la résistance très importante du virus aphteux dans le milieu extérieur (Leforban, 2003). Le virus de la FA peut d'autre part se propager sur de longues distances par voie aérienne (Donaldson et Alexandersen, 2002).
Les résultats ont montré que tous les type d'élevages étaient affectés par la FA, y compris les élevages intensifs comme les systèmes laitiers fermés où la biosécurité est maîtrisée. Pourtant, le mode d'élevage extensif paraît le plus à même de favoriser la transmission du virus, car on y observe un risque plus important de mélange de différents troupeaux et de contacts étroits entre animaux d'origines diverses, associé à la faible disponibilité de l'eau et de l'alimentation. Le risque de propagation de la maladie est surtout important pendant la saison sèche au cours de laquelle les animaux se rassemblent dans les pâturages et aux points d'eau.

« Le virus de la FA est virus à ARN qui, en raison de réplication impliquant une ARN polymérase ARN-débendante sède un taux de mutation assez élevé de $10^{-3}$ à $10^{-4}$ parntucléotide par cycle de réplication de l'ARN » (Thiry et al, 2001). «Ces mutations génèrent des quasi-espèces qui favorisent la multiplicité antigénique » (Brehmet al., 2008). «L'immunité croisée entre les sous-types au sein d'un même type n'est souvent que partielle » (Geering, 1986) et « l'introduction d'un nouveau type antigenique dans un troupeau peut entraîner une morbidité pouvant-aller jusqua à $80 \%$ dans les zones endémiques » (Houndjè et al, 2013). Ainsi, un animal vacciné envers un sérotype de virus peut presenter après la vaccination un tableau clinique de FA dû à un autre sous-type du même sérotype, ou à un autre sérotype viral. Malgré cela, la différence de séroprévalence constatée dans notre étude entre les animaux vaccinés et non vaccinés a été significative parce que l'infection due uniquement aux sérotypes $\mathrm{O}$ et $\mathrm{A}$, présents dans le vaccin, représentait $54,2 \%$ des infections.

Parmil les sept sérotypes immunologiques distincts qui circulent dans le monde, cinq ont été identifiés en Algérie à travers cette enquête. Outre les sérotypes $\mathrm{A}$ et $\mathrm{O}$ déjà identifiés et contre lesquels des campagnes de vaccination ont été mises en place, trois autres sérotypes (SAT SAT 2 et Asia 1) ont été mis en évidence, ce qui indique qu'il y areu de nouvelles introductions du virus aphteux en Algérie. Or, les sérotypes SAT 1 et SAT 2 ne circulent ni en Tunisie ni en Libye. L'introduction serait plutôt liée à la transhumance de zébus en provenance des pays d'Afrique subsaharienne (ASS), qui jouerait ainsi un rôle significatif au plan épidémiologique pour les ruminants d'Algérie. En effet, la transhumance interpays constitue une voie prépondérante de transmission de la FA en ASS (Bizimana, 1994), la FA étant enzootique dans presque tous les pays d'ASS (Souley Kouato et al., 2018) où la lutte contre cette maladie est plus compliquée que dans les autres régions du monde. La FA est endémique dans cette région et pourtant « très peu de cas de fièvre aphteuse sont rapportés en raison soit du système d'élevage qui est de type extensif, soit par manque de sensibilisation des populations locales, soit par manque de moyens et de volonté politique des gouvernements de cette région » (Garabed et al., 2009).

Pour lutter contre la FA, l'Algérie a choisi d'appliquer la vaccination avec un vaccin bivalent (sérotypes $\mathrm{A}$ et $\mathrm{O}$ ), la séquestration des foyers et l'abattage des animaux malades. Un bon nombre de pays qui sont parvenus à contrôler la FA ont également appliqué des mesures sanitaires strictes et/ou eu recours à la vaccination (Paton et al., 2009). Il est important de noter que la FA a été éradiquée dans de vastes régions (près de 40 pays concernés), comme l'Europe de l'Ouest qui a cessé de vacciner après une dizaine d'années de campagnes massives ininterrompues. L'Amérique du Nord, la majeure partie des pays d'Amérique latine et ceux du Pacifique Sud sont aujourd'hui également indemnes (Domenech et Vallat, 2012). Toutefois, l'absence actuelle presque totale de vaccination en ASS, et plus particulièrement en Afrique de l'Ouest (Vosloo et al., 2002), ne favorise pas le contrôle de la maladie. Par ailleurs, l'application de mesures sanitaires très sévères, comme l'abattage des animaux présents dans les foyers, l'abattage en anneau des élevages proches ou ayant eu des contacts à risque avec les élevages infectés, les restrictions à la circulation des animaux et des produits d'origine animale, semble irréaliste dans les 
pays d'ASS en raison du manque de moyens financiers et du faible intérêt accordé par les gouvernements à la surveillance et à la lutte contre les maladies contagieuses des animaux. C'est pourquoi, en matière de surveillance de la FA l'autorité vétérinaire algérienne compte sur le programme d'identification du cheptel et l'installation des observatoires au sud du pays qui, une fois achevés, permettraient d'assurer une détection plus précoce des maladies transsahariennes et un contrôle des mouvements du cheptel.

\section{CONCLUSION}

L'enquête a permis d'estimer la séroprévalence actuelle et le taux de couverture vaccinale de la fièvre aphteuse dans plusieurs wilayas de l'ouest de l'Algérie. Elle a mis en évidence quelques facteurs qui contribuent au maintien de cette maladie dans la région, comme la variabilité antigénique et la propagation du virus aphteux, mais aussi l'efficacité de vaccin antiaphteux. Parmi les systèmes d'élevage en vigueur dans le pays, aucun ne présente de risque plus important d'atteinte par la FA. Les animaux non vaccinés ont en revanche présenté un risque plus important de contracter la FA que les animaux vaccinés. La moitié de la population étudiée avait reçu un vaccin bivalent A et $\mathrm{O}$ mais l'efficacité du vaccin pourrait encore être améliorée si le cocktail vaccinal était modifié. Comme de nombreux pays d'Europe et d'Amérique, l'Algérie pourrait passer au statut de pays indemne avec vaccination si les campagnes de vaccination étaient pratiquées sans interruption chez les bovins et les petits ruminants, quel que soit le mode d'élevage (extensif, intensif ou semi-intensif), en adaptant la composition du vaccin à la nature des souches circulant dans le pays, notamment en intégrant les sérotypes SAT 1 et Asia 1, surtout pour les bovins qui se sont révélés être les plus touchés.

\section{Conflits d'intérêts}

La présente enquête a été réalisée sans conflit d'intérêts. II s'a travail de recherche dont la finalité est l'estimation matière de fièvre aphteuse dans la région étudiée.

\section{Déclaration des contributions des auteurs}

$\mathrm{AB}$ a analysé et interprété les données et résultats, rédigé la première version du manuscrit ; AK a planifié l'enquête et la fourniture des kits Elisa, réalisé les prélèvements sanguins, collecté les données ; AS a géré l'équipe des techniciens d'analyse :MK aréalisé les analyses statistiques des résultats, TMH et DKont fait la révision critique du manuscrit.

\section{REFERENCES}

Alexandersen S., Zhang Z., Donaldson A.I., Garland A.I.M., 2003. The pathogenesis and diagnosis of foot-and-mouth disease. J. Comp. Pathol.,129 (1): 1-36, doi: 10.1016/s0021-9975(03)00041-0

Bizimana N., 1994. Epidemiology, surveillance and control of the principal infectious animal diseases in Africa. Rev. Sci, Tech. Off. Int. Eplzoot., 13 (2): 397-416, doi: 10.20506/rst.13.2.770

Bouguedour R., 2017. Analyse de la situation au regard de la fièvre aphteuse et de la peste des petits ruminants en Afrique en 2015 et 2016. Atelier régional OIE fièvre aphteuse et peste des petits ruminants Tunis (Tunisie), 14-16 mars 2017. https://r-africa oie.int/wp-content/uploads/2017/03/oiefmd-and-ppr-in-africa.pdf (consulté 30jan 2021)

Brehm K.E., Kumar Thulke H.H.. Haas B., 2008. High potency vaccines induce protection against heterologous challenge with foot-and-mouth disease virus. Vaccine, 26 (13): 1681-1687, doi: 10.1016/j.vaccine.2008.01.038

Calavas D, Gauchard J., Hendrikx P., Lambert Y., Mercier A., Kassimi L.B., 2018 Fièvre aphteuse en Algérie. Point de situation au 31 Juillet 2018, www.plateforme-esa.fr/article/fievre-aphteuse-en-algerie-point-de-situation-au-31 juillet-2018 (consulté 20 déc. 2020)

Domenech Vallat B., 2012. La lutte contre les épizooties au XXI siècle. $C R$ Biol, 335 (5).356-369, doi: 10.1016/j.crvi.2012.04.003

Donaldsen A.1., Alexandersen S., 2002. Predicting the spread of foot and mouth disease by airborne virus. Rev. Sci. Tech. Off. Int. Epizoot., 21 (3): 569-575, doi: 10.20506/rst.21.3.1362

Garabed R.B., Perez A.M., Johnson W.O., Thurmond M.C., 2009. Use of expert opinion for animal disease decisions: an example of foot-and-mouth disease status designation. Prev. Vet. Med., 92 (1-2): 20-30, doi: 10.1016/j. prevetmed.2009.06.010

Geering W.A., 1986. Maladies prioritaires du bétail. FAO, Rome, Italie, 421 p.

Houndjè E., Kpodékon M., Moutou F., Blaise-Boisseau S., Bakkali-Kassimi L., Berkvens D., Zientara S., et al., 2013. Principales caractéristiques épidémiologiques et impact économique de la fièvre aphteuse en Afrique : synthèse bibliographique. Ann. Méd. Vét., 157: 120-134
Leforban Y., 2003. Fièvre aphteuse. In : Principales maladies infectieuses et parasitaires du bétail. Europe et régions chaudes. Tome 1 : Généralités ; maladies virales (Eds : Lefèvre P.C., Blancou J., Chermette R.). Tec \& Doc Lavoisier, Paris, France, 339-361

Maree F.F., Kasanga C.J., Scott K.A., Opperman P.A., Chitray M., Sangula A.K., Sallu R., et al., 2014. Challenges and prospects for the control of footand-mouth disease: an African perspective. Vet. Med. Res. Rep., 5: 119 138, doi: 10.2147/VMRR.S62607

OIE, 2018a. Récents foyers et événements épidémiologiques en Afrique. https://rr-africa.oie.int/fr/notifications-immediates-en-afrique/ (consulté 27 juin 2021

OIE, 2018b. Récents foyers et événements épidémiologiques en Afrique. www.oie.int/fr/maladie/fievre-aphteuse/ (consulté 27 juin 2021)

OIE, 2018c. Manuel des tests de diagnostic et des vaccins pour les animaux terrestres 2019. www.oie.int/fileadmin/Home/fr/Health_standards/ tahm/3.01.08_FMD.pdf (consulté 30 déc. 2020)

Paton D., Sumption K., Charleston B., 2009. Options for control of foot-andmouth disease: knowledge, capability and policy. Philos. Trans. R. Soc. B. 364: 2657-2667, doi: 10.1098/rstb.2009.0100

Souley Kouato B., De Clercq K., Abatih E., Dal Pozzo F., King D.P., Thys E., Marichatou $\mathrm{H}_{\text {., }}$ et al., 2018. Review of epidemiological risk models for footand-mouth disease: Implications for prevention strategies with a focus on Africa. PLoS One, 13 (12): e0208296, doi: 10.1371/journal.pone.0208296

Thiry E., Baranowski E., Domingo E., 2001. Epidémiologie moléculaire de la fièvre aphteuse. Epidémiol. Santé Anim., 39: 59-67

Vosloo W., Bastos A.D.S., Sangare O., Hargreaves S.K., Thomson G.R., 2002 Review of the status and control of foot and mouth disease in sub-Saharan Africa. Rev. Sci. Tech. Off. Int. Epizoot., 21: 437-449, doi: 10.20506/ rst.21.3.1349 


\section{Summary}

Bouziri A., Sebai A., Kebir A., Khames M., Hamdi T.M., Khelef D. Foot-and-mouth disease in cattle and small ruminants in Algeria. Seroepidemiological survey in the western region

The foot-and-mouth disease (FMD) is an infectious disease of viral origin that affects ruminants and pigs. A seroepidemiological survey was conducted in ruminants in the western region of Algeria to estimate the seroprevalence of FMD and to determine the risk factors that might lead to animals being affected by this disease. A total of 420 sera were collected (210 from cattle and 210 from small ruminants) and subjected to an NSP ELISA for the detection of antibodies against FMD virus induced by nonstructural proteins, followed by ELISAs for the detection of structural proteins specific to serotypes A, O, SAT 1, SAT 2 and Asia 1. The results showed a non-significant difference ( $p>0.05)$ in seroprevalence between species with $23.8 \%$ in cattle and $27.1 \%$ in small ruminants. Serotype O was present in $95.3 \%$ of positive sera. It was present singly in $64.9 \%$ of small ruminants, whereas cattle were usually infected with several serotypes simultaneously, with the predominant association of the four serotypes A, O, SAT 1 and Asia 1, which accounted for $36 \%$ of infections. Vaccination coverage reached $48.7 \%$ of the herd (cattle and small ruminants) over six months of age. No statistical association was found between the farming system (extensive, semi-intensive or intensive) and FMD infection ( $p>0.05)$. In contrast, a significant association $(p<0.05)$ was found between animal vaccination and infection, with vaccinated animals being less infected with FMD.

Keywords: cattle, small ruminants, foot-and-mouth disease morbidity, Algeria

\section{Resumen}

Bouziri A., Sebai A., Kebir A., Khames M., Hamdi T.M., Khelef D. Fiebre aftosa en los bovinos y pequeños rumiantes en Argelia. Encuesta sero-epidemiológica en la región del oeste

La fiebre aftosa (FA) es una enfermedad infecciosa de origen viral, que afecta los rumiantes y los porcinos. Una encuesta sero-epidemiológica se llevó a cabo en los rumiantes de lá regiốn oeste de Argelia, con el fin de estimar la seroprevalencla de la FA y de determinar los factores de riesgo susceptibles de favo recer la aparición de esta patología en los animales. En total se recolectaron 420 sueros (210 de bovinos y 210 de pequeños rumiantes) y se sometieron a la prueba Elisa NSP, permitiendo la detección de anticuerpos dirigidos contra el virús de la FA inducidos por las proteínas no estructurales, Ruego a pruebas de Elisa para detectar las proteínas estructurales específicas de los serotipos A, O, SAT 1, SAT 2 y Asia 1. Los resultados mostraron una diferencia no significativa $(p>0,05)$ de la seroprevalencia entre las especies con $23,8 \%$ en los bovinos y $27,1 \%$ en los pequeños rumiantes. El serotipo O estaba presente en $95,3 \%$ de los sueros positivos. Estaba presente en exclusividad en $64,9 \%$ de los pequeños rumiantes, mientras que los bovinos estaban generalmente infectados simultáneamente por varios serotipos, con la asociación predoninante dé cuatro serotipos A, O, SAT 1 y Asia 1, gue representaron $36 \%$ de las infecciones. La tasa de cobertura de vacunación alcanzó $48,7 \%$ del hato (bovinos y pequeños rumiantes) con edades mayores de seis meses. No idenció ninguna relación estadística entre el tipo de cría xtensiva, semi intensiva o intensiva) y el padecimiento por FA $(p>0,05)$. Por otro lado, una asociación significativa $(p<0,05)$ fue establecida entre la vacunación de los animales y la infección, Ios anımales vacunados fueron menos infectados por la FA. Palabras clave: bovino, pequeños rumiantes, fiebre aftosa, morbosidad, Argelia 